\title{
Different strategies from monotherapies to dual or triple fixed dose combination therapies to achieve blood pressure goals: a summary of a satellite symposium from the European Society of Hypertension
}

\author{
Massimo Volpe ${ }^{1}$
}

Published online: 8 July 2015

(c) Springer International Publishing Switzerland 2015

In this special issue of High Blood Pressure and Cardiovascular Prevention we report the proceedings of a satellite symposium at the European Society of Hypertension (ESH) held in Athens, Greece in June 2014. The symposium, entitled "Effective fixed solutions: one, two, three... goal!", addressed a major issue in the contemporary management of hypertension, that is, how to overcome the poor control of hypertension in patients and, more in general, in the population.

Professor Josep Redòn from Spain provides the introductory remarks in this special issue that outline the current topics in the field of hypertension while another key opinion leader, Professor George Bakris, from Chicago in the United States, provides summary statements about where the field of hypertension is heading. The two keynote papers within this special issue reflect on current clinical data and identify potential areas for improvement for managing BP control in patients with hypertension.

The topic on how to achieve BP goal in hypertension by Professor Stéphane Laurent from France, is a competent and updated review on the role of combination therapy in the guidelines and in recent studies, and proposes an approach of "personalized" hypertension therapy platform which has also been recently proposed by a panel of international experts.

Massimo Volpe

Massimo.volpe@uniroma1.it

1 Division of Cardiology, Department of Clinical and Molecular Medicine, Faculty of Medicine and Psychology, University of Rome "Sapienza", Sant'Andrea Hospital, Rome, Italy
The second paper by Professor Roland Schmieder from Germany focuses on the issue of treatment-resistant hypertension and is a practical, synthetic approach to this topic of growing interest for the medical community. The diagnosis of true resistant hypertension represents an excellent exercise to put in practice clinical guidelines and good clinical practice in the diagnostic process.

Finally, two clinical cases entitled "A patient with apparent compliance" and "A patient with apparent resistant hypertension" by Dr. Sarafidis and Dr. Aguiar, respectively, provide practical insights for the readers of this journal who are interested in these subject matters.

Single-pill combination therapy based on one, two or three drugs represent a truly new strategy to fight the unmet needs in the control of hypertension, and the title of this symposium, while proposing this new therapeutic solution with a metaphoric soccer representation, reflects the current challenges and the proposed solutions.

As the Editor of the Journal, I am honoured and proud to host the presentations of these scientific leaders in the field of hypertension.

Massimo Volpe, M.D, FESC, FAHA

Editor-in-Chief

High Blood Pressure and Cardiovascular Prevention 